\title{
Studiengang
}

Wolfgang Semar*

\section{Master of Science in Business Administration - Major Information and Data Management}

https://doi.org/10.1515/iwp-2020-2107

Die Fachhochschule Graubünden (FHGR) bietet durch das Schweizerische Institut für Informationswissenschaft (SII), in seiner Eigenschaft als akademische Ausbildungsinstitution, seit 2010 einen konsekutiven Masterstudiengang an. Der Studieninhalt dieses Masterstudiengangs hat sich seit seinem Start drei Mal geändert, um dem jeweiligen Marktbedürfnis Rechnung zu tragen. Im folgenden Beitrag skizzieren wir die Entwicklung und beschreiben das aktuelle Lehrangebot des konsekutiven Masterstudiengangs Information and Data Management.

\section{Master of Science in Information Science (MSc IS)}

Mit dem Herbstsemester 2010 startete der damalige Master of Science in Information Science (MSc IS) als eigenständiger Studiengang mit 33 Studierenden. Die Studiendauer wurde von den üblichen drei Semestern auf vier Semester angehoben. So wurde erreicht, dass die Anzahl ECTS pro Semester geringer ist als die üblichen 30. Dies hat den Vorteil, dass der Präsenzunterricht auf zwei Tage in der Woche (freitags und samstags) verteilt werden kann. Durch diese Verlängerung der Studiendauer konnten Berufstätige mit einem Arbeitspensum bis zu 60 Prozent am Studium teilnehmen. Dieser Trick war notwendig, da unsere Zielgruppe vornehmlich bereits berufstätig war. Sie bestand größtenteils aus Absolventinnen und Absolventen des Bachelorstudiengangs Informationswissenschaft sowie dem früheren Diplomstudiengang Information und Dokumentation der Hochschule für Technik und Wirtschaft (HTW) Chur. Speziell für diese Zielgruppe wurde auch der Studieninhalt konzipiert. Neben Modulen zu Wissenschaftsund Forschungstheorien sowie speziellen Information-Sci-

*Kontaktperson: Prof. Dr. habil. Wolfgang Semar, Fachhochschule Graubünden, Schweizerisches Institut für Informationswissenschaft, Pulvermühlestrasse 57, 7000 Chur, Schweiz,

E-Mail: wolfgang.semar@fhgr.ch
ence-Modulen, an denen alle Studierenden teilnehmen, standen zwei Vertiefungsrichtungen zur Auswahl. In der Vertiefung Information Asset Management wurden innovative Bibliotheks- und Archivthemen wie Management digitaler Bibliotheken, Open Access und Records Management gelehrt, während die zweite Vertiefung Information Design Module wie Methoden der Web-Anwendungsentwicklung, Wissensrepräsentation sowie Design und Evaluation von Benutzeroberflächen beinhaltete. Zusätzlich hatten die Studierenden die Möglichkeit, an unseren Partnerhochschulen Wahlmodule zu belegen. Mit diesem Konzept wurde im Hebst 2012 der Studiengang ein zweites Mal durchgeführt, allerdings diesmal nur mit 28 Studierenden. Eine Umfrage bei der damaligen Zielgruppe ergab, dass wir bereits die meisten Interessierten erreicht hatten und wir beim nächsten Start mit viel Glück auf eine Teilnehmendenzahl von um die 15 Personen kommen würden.

\section{Master of Science in Business Administration (MSc BA) - Major Information and Data Management}

Unter diesen Bedingungen hatte sich die Hochschulleitung dazu entschlossen, den Studiengang mit zwei weiteren konsekutiven Masterstudiengängen der Hochschule zusammenzulegen. Dieses neue Konstrukt sah vor, dass die Informationswissenschaft lediglich eine Vertiefung, mit der neuen Bezeichnung Information and Data Management, von insgesamt drei Vertiefungen eines Studiengangs Master of Science in Business Administration (MSc BA) ist. Der grundlegende Aufbau des neuen Studiengangs wurde jedoch vom MSc IS übernommen. Wieder wurden 90 ECTS auf vier Semester verteilt, da sich dieses Konzept als sehr sinnvoll erwiesen hatte. Ebenso mussten alle Studierenden die Module des Wissenschaftlichen Arbeitens sowie General-Management-Module besuchen. Jedoch wurde der Inhalt der Vertiefung Information and Data Management völlig umgestaltet und an die zukünftige Marktsituation angepasst. Neue Entwicklungen und Paradigmenwechsel im Internet wie Cloud-Dienste, Mobile Computing 
und Data Science wurden frühzeitig aufgegriffen und im neuen Curriculum umgesetzt. Das Ziel war, Informationswissenschaftlerinnen und -wissenschaftler auszubilden, die in der Lage sind, heterogene Datenmengen zu analysieren und zu bearbeiten, um für die Nutzenden einen Mehrwert zu schaffen. Im Einzelnen kamen folgende Module zum Einsatz: Advanced Web Technologies, Introduction to Data Management and Analysis, Collaborative Knowledge Management, Knowledge Representation and Semantic Technologies, User Interface Design, Advanced Information Retrieval, Information Security, Information Visualisation, Advanced Topics of Information Science und Trends in Data Management. Mit diesem Curriculum wurde der Masterstudiengang von 2013 bis 2018 jährlich erfolgreich mit 20 bis 25 Studierenden gestartet. Seit 2018 werden aber von Schweizer Universitäten und Fachhochschulen massiv neue Data-Science-Studiengänge angeboten. Dies führte dazu, dass die Anmeldezahlen für unseren Studiengang im Jahr 2019 auf 15 Neustudierende zurück ging. Dieser Umstand und die geänderte akademische Ausbildungssituation bewogen uns dazu, ab dem Herbstsemester 2020 ein neues Curriculum anzubieten.

Damit folgen wir nicht dem derzeitigen Data-ScienceTrend, sondern wagen einen weiteren Schritt in die $\mathrm{Zu}$ kunft der Informationswissenschaft. Mit dem Fokus auf die nutzergerechte Visualisierung großer Datenmengen haben wir schweizweit ein Alleinstellungsmerkmal in der akademischen Ausbildung erreicht. Dass wir zukünftig mehr und mehr mit Daten, seien es strukturierte Daten wie Produktionsdaten in Unternehmen, Nutzungsdaten von Apps und Webshops sowie unstrukturierte Daten wie Texte, Bilder, Musik und Videos aus Web-Anwendungen zu tun haben, ist unbestritten. Aber gerade diese Datenüberflutung erfordert Expertinnen und Experten, die verschiedene Verfahren, Technologien und Instrumente zur Datenaufbereitung als Grundlage beherrschen, aber darüber hinaus in der Lage sind, Visualisierungsmethoden und -techniken benutzergerecht so ein- und umzusetzen, dass daraus eine Information wird, ganz im Sinne des Kuhlenschen „pragmatischen Primats“ (Kuhlen 2013, S. 3). Dieses Curriculum baut auf Information Science- und DataScience-Kenntnissen auf und erweitert diese durch eine Visualisierungskompetenz. Denn nur in dieser Kombination kann tatsächlich aus Daten Information und damit wieder die Grundlagen für neues Wissen geschaffen werden.

\section{Modularer Studienaufbau}

Die Studieninhalte verteilen sich auf vier Semester. Dies ermöglicht berufstätigen Studierenden ein Arbeitsverhält- nis von bis zu 60 Prozent, was, wie sich gezeigt hat, für unsere Klientel ein entscheidender Vorteil ist. Bei diesem, ab Herbst 2020 startendem, Curriculum gab es aber noch weitere Veränderungen. So wurden unterschiedliche Modultypen wie Wahl-, Wahlpflicht- und Pflichtmodule eingeführt. Pflichtmodule sind obligatorisch zu absolvieren, Wahlpflichtmodule können aus einem vorgegebenen Bereich in einem festgelegten Umfang ausgewählt werden und Wahlmodule sind aus einem umschriebenen Bereich frei wählbar. Durch die Wahlmöglichkeiten haben die Studierenden nun den Vorteil einen Teil ihrer Module ganz nach ihren eigenen Wünschen, Fähigkeiten und Zielen zusammenzustellen. Die unterschiedlichen Module sind inhaltlich und zeitlich abgeschlossen. Sie können sich aus einer oder mehreren Lehrveranstaltungen, sogenannten Kursen, zusammensetzen. Module erstrecken sich in der Regel über ein oder zwei Semester. Diese Flexibilität in der Wahl der Module ist sicherlich ein weiterer Pluspunkt dieses Curriculums. Im Folgenden werden wir den derzeitigen Aufbau des Studiums, wie es im Herbstsemester 2020 starten wird, erläutern.

Im ersten Semester müssen alle Studierenden am Pflichtmodul Research Methods teilnehmen. Die drei Kurse in diesem Modul vermitteln, wie praxisrelevante Probleme auf wissenschaftlich fundierte Weise gelöst werden. Der Kurs Research Design zeigt, wie man anspruchsvolle Projekte vorbereitet und abwickelt. Die Kurse Qualitative und Quantitative Research Methods demonstrieren, wie man mit qualitativen und quantitativen Untersuchungsmethoden neue Erkenntnisse gewinnt. Als weitere Pflichtveranstaltungen müssen die Studierenden $P y$ thon for Data Scientists und Introduction to Data Science besuchen. In den ersten drei Semestern haben die Studierenden die Möglichkeit aus insgesamt zehn General-Management-Modulen fünf auszuwählen. Im Einzelnen sind dies: Corporate Communication, Corporate Responsibility, Sustainability Management, Digital Business, Leading Change, Organisational Development, Organisational Leadership, Talent Management, Introduction to Data Management und Fundamentals of Web Data Analytics. Für den Major Information and Data Management empfehlen wir für fachfremde Studierende, die Module Introduction to Data Management und Fundamentals of Web Data Analytics zu wählen, um so einen Einstieg in die Datenthematik zu erhalten. Um die Vertiefung Information and Data Management abzuschließen müssen die Studierenden weitere Pflichtmodule belegen. Sie werden mit grundlegenden Konzepten zur Beschreibung von Struktur, Sprache und Schritten zu deren maschineller Bearbeitung vertraut gemacht und erlernen Werkzeuge und Techniken, um Daten in größerem Umfang und aus unterschiedlichen 
Anwendungsbereichen zu visualisieren. Sowohl betriebswirtschaftliche als auch wissenschaftliche Datenquellen und Visualisierungen werden behandelt.

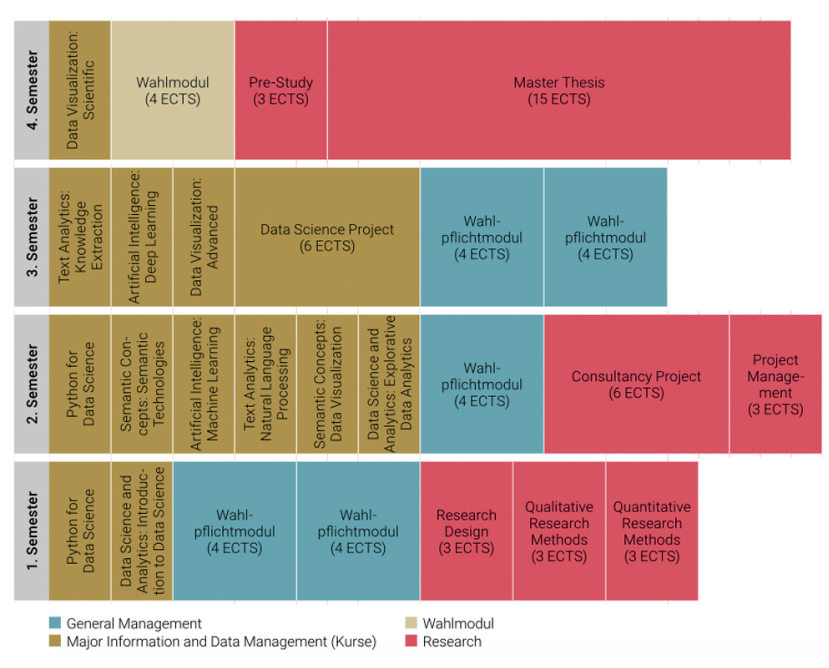

Abbildung 1: Aufbau des Curriculums Masterstudiengang Major Information and Data Management ab Herbst 2020.

Als besondere Dienstleistung werden die Vorlesungen live per Video via Internet übertragen und stehen den Studierenden anschließend als abgespeicherte Dateien zwecks Vertiefung im Selbststudium zur Verfügung. Dennoch handelt es sich bei diesem Studium nicht um ein Fernstudium. Das Zusammenspiel zwischen Studium, Forschung und Praxis ist ein wichtiger Aspekt. Die Fachhochschule ist vom Bund und den Kantonen anerkannt, sodass ein Anspruch auf Stipendien geltend gemacht werden kann. Die Fachhochschule Graubünden nimmt zudem am Erasmusprogramm der EU teil.

\section{Zukunftsperspektiven}

Durch die digitale Transformation werden zukünftig in allen Bereichen neue Daten im Sekundentakt anfallen. Die globale und interdisziplinäre Verknüpfung und Auswertung dieser Daten erfordert Expertinnen und Experten, die die neuesten Verfahren, Instrumente und Technologien beherrschen, um die in den Daten enthaltene Information $\mathrm{zu}$ extrahieren und in anspruchsvollen Beratungs- und Managementprojekten umzusetzen. Die vielfältigen Aufgabenbereiche und Ausrichtungen der Studienrichtung eröffnen den Absolventinnen und Absolventen ein breitgefächertes Beschäftigungsfeld in den unterschiedlichsten Branchen.
Deskriptoren: Studium, Business Administration, Data Sience, Informationswissenschaft, Fachhochschule

\section{Literatur}

Kuhlen, R. (2013). Information - Informationswissenschaft. In: Rainer Kuhlen, Wolfgang Semar, Dietmar Strauch (Hrsg.): Grundlagen der praktischen Information und Dokumentation. 6. Ausgabe. Walter de Gruyter, Berlin.

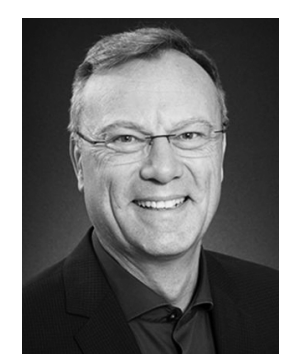

Prof. Dr. habil. Wolfgang Semar

Fachhochschule Graubünden

Schweizerisches Institut für

Informationswissenschaft

Pulvermühlestrasse 57

7000 Chur

Schweiz

wolfgang.semar@fhgr.ch

Nach Abschluss seines Studiums des Wirtschaftsingenieurwesens an der Universität Kaiserslautern erwarb Wolfgang Semar das Diplom im Aufbaustudium der Informationswissenschaft an der Universität Konstanz bei Prof. Kuhlen, wo er promoviert wurde und sich habilitierte. Von 2006 bis 2008 vertrat er den Lehrstuhl Informationswissenschaft an der Universität des Saarlandes. Seit 2008 ist er Leiter des Majors Information and Data Management am konsekutiven Masterstudiengang der Fachhochschule Graubünden. Seine derzeitige Forschungstätigkeit liegt im Gebiet des kollaborativen Wissensmanagements. 